\title{
CVD-DIAMOND-BASED POSITION SENSITIVE PHOTOCONDUCTIVE DETECTOR FOR HIGH-FLUX X-RAYS AND GAMMA RAYS
}

\author{
Deming Shu, P. K. Job, Juan Barraza, Tim Cundiff, and Tuncer M. Kuzay, \\ Advanced Photon Source, Argonne National Laboratory, \\ 9700 S. Cass Av. Argonne, IL 60439, U.S.A.
}

\section{Abstract}

A position-sensitive photoconductive detector (PSPCD) using insulating-type CVD diamond as its substrate material has been developed at the Advanced Photon Source (APS). Several different configurations, including a quadrant pattern for a x-ray-transmitting beam position monitor (TBPM) and 1-D and 2-D arrays for PSPCD beam profilers, have been developed. Tests on different PSPCD devices with high-heat-flux undulator white $\mathrm{x}$-ray beam, as well as with gamma-ray beams from ${ }^{60} \mathrm{Co}$ sources have been done at the APS and National Institute of Standards and Technology (NIST). It was proven that the insulating-type CVD diamond can be used to make a hard x-ray and gamma-ray positionsensitive detector that acts as a solid-state ion chamber. These detectors are based on the photoconductivity principle.

A total of eleven of these TBPMs have been installed on the APS front ends for commissioning use. The linear array PSPCD beam profiler has been routinely used for direct measurements of the undulator white beam profile. More tests with hard x-rays and gamma rays are planned for the CVD-diamond 2-D imaging PSPCD. Potential applications include a high-dose-rate beam profiler for fourth-generation synchrotron radiation facilities, such as free-electron lasers.

\section{INTRODUCTION}

Natural diamonds as photoconductive radiation detectors (PCDs) have been studied since 1956 [1], and only certain diamonds, those with low impurity concentrations (specifically nitrogen), were found to be suitable for use as radiation detectors [2]. Natural diamonds have been used as PCDs for soft x-ray detection with a laser-produced plasma soft $\mathrm{x}$-ray source and a synchrotron radiation source [3]. Insulating type (type IIa) synthetic diamonds (from a high-pressure cell) used as solid-state ionization-chamber radiation detectors have been studied for biological applications with alpha particles and gamma radiation [4]. Compared with other photoconductors, diamond is a robust and radiationhardened material with high dark resistivity and a large breakdown electric field; diamond is also sensitive to hard X-rays [5].

The working principle of a position-sensitive photoconductive detector (PSPCD) can be described as follows: a thin CVD-type diamond disk is patterned on both surfaces with a thin layer of electrically conductive material, such as aluminum, etc. These coated patterns are individually connected to a biased current-amplifier circuitry through an ohmic contact. When the electrically biased CVD disk is subjected to either monochromatic or white $\mathrm{x}$-ray beam, the photons activate the impurities in the CVD diamond causing a local conductivity change, then a local current change through the contact points. The amount of the generated current is a function of the photon flux.

\section{PSPCD TESTED AT THE APS WITH X-RAYS}

A compact filter/mask/window assembly has been designed for undulator beamline commissioning activity at the APS beamlines [6]. The assembly consists of one $300-\mu \mathrm{m}$ graphite filter, one $127-\mu \mathrm{m}$ CVD diamond filter and two $250-\mu \mathrm{m}$ beryllium windows. A water-cooled Glidcop fixed mask with a $4.5 \mathrm{~mm}$ X $4.5 \mathrm{~mm}$ output optical aperture and a $0.96 \mathrm{mrad}$ X $1.6 \mathrm{mrad}$ beam missteering acceptance is a major component in the assembly. The CVD diamond filter is mounted on the downstream side of the fixed mask and is designed to also function as a transmitting hard $\mathrm{x}$-ray beam position monitor. It has a quadrant pattern configuration. From the test results, we have learned that, compared to a photoemmision-type TBPM, the beam position signal from a photoconductive-type TBPM has less undulator gap dependence [7].

A x-ray-transmitting beam profiler system using two linear-array PSPCDs has been designed for Advanced Photon Source (APS) undulator beamline commissioning [8]. The same insulating-type CVD diamond disk was used as for the linear array substrate. On each disk, sixteen $0.2-\mu \mathrm{m}$-thick, $175-\mu \mathrm{m}$-wide aluminum strips were coated on one side, and an orthogonal single $175-\mu \mathrm{m}$-wide strip was coated on the other side. Hence looking through the disk, a linear array of sixteen pixels was created as the 
photoconductive sensor elements, with $175 \mu \mathrm{m}$ x $175 \mu \mathrm{m}$ pixel size.

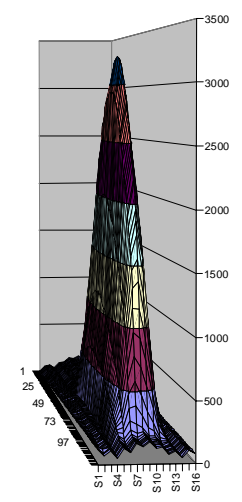

Fig. 1. A typical profile of APS undulator white beam directly measured by a 16-pixel linear-array PSPCD.

During the measurement, two sets of 16-pixel linear array PSPCDs are placed perpendicular to each other into the hard x-ray beam.. The two arrays read out the beam's vertical and horizontal profile information simultaneously. To obtain a complete beam 2-D profile, one can scan across the beam. Fig. 1 shows a typical profile of an APS undulator white beam directly measured by a 16-pixel linear-array PSPCD scanning across the beam with the undulator magnet gap setting equal to $15 \mathrm{~mm}$. A $12.7-\mathrm{mm}$-thick aluminum filter was used for these measurements to eliminate most of the soft $\mathrm{x}$-rays.

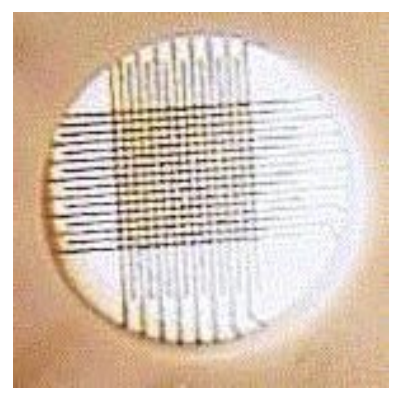

Fig. 2, Sixteen aluminum strips are coated on both sides of the CVD-diamond disk creating a $16 \times 16$ pixel twodimensional array with $175 \mu \mathrm{m} \times 175 \mu \mathrm{m}$ pixel size.

A prototype of a 2-D imaging PSPCD has been built at the APS. As shown in Fig. 2, sixteen aluminum strips are coated on both sides of the CVD-diamond disk creating a $16 \times 16$ pixel two-dimensional array with a $175 \mu \mathrm{m} \times 175 \mu \mathrm{m}$ pixel size. The CVD-diamond disk was mounted on a water-cooled base with a couple of ceramic connector-interface disks. Preliminary tests proved that a 2-D hard $\mathrm{x}$-ray beam profile image could be read out by a multichannel current amplifier with pulsed bias electronics.

Fig. 3 shows the schematic of the readout electronics for the prototype of 2-D imaging PSPCD. Two sets of multichannel analog switches are synchronized by a clock, which also provides the triggering signal for the computer data acquisition system. For each scanning position, one of the vertical strips was pulsed by a bias-voltage, and the 16-channel current amplifier then read out the current signal from the identical column of the imaging pixels on the CVDdiamond disk. A 16 x 16 LED array was used as an imaging screen. The scanning rate was $300-3000$ columns per second. A steady 2-D image was observed with the APS undulator white beam.

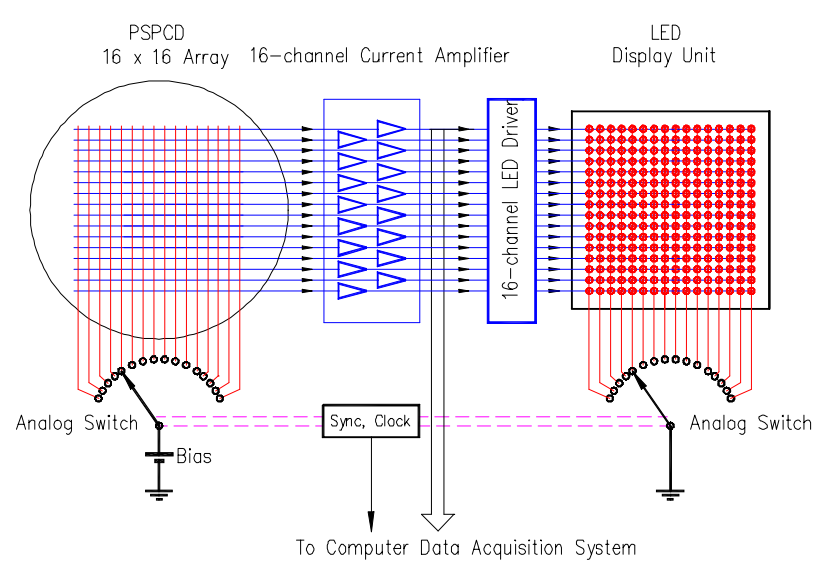

Fig. 3. Schematic of the readout electronics for the prototype of the 2-D imaging PSPCD.

We have tested the single-pixel response of this 2D imaging PSPCD using undulator white beam with a $150 \mu \mathrm{m} \times 150 \mu \mathrm{m}$ aperture. It was found that the pixels in this 2-D array PSPCD do not cross talk. Fig. 4 shows different images observed on the LED array with variant beam condition and slit settings. We have also studied several different CVD-diamond products from different vendors with different manufacturing process. We found that, only certain CVD-diamonds, those with low impurity concentrations (specifically nitrogen and graphite), are suitable for use as imaging detectors.

\section{TESTS OF THE PSPCD WITH GAMMA RAYS}

We have started study of the CVD-diamond-based position sensitive detector with high-dose-rate gammaray beams. Preliminary experimental results with a ${ }^{60} \mathrm{Co}$ source at a NIST radiation laboratory show that it is 
feasible to use CVD-diamond as an imaging detector for high-dose-rate gamma rays.

The high-flux ${ }^{60} \mathrm{Co}$ vertical-beam source, which we used at the NIST, has 33.0 Gray/hour or $1.45 \times 10^{9}$ photons $/ \mathrm{cm}^{2} / \mathrm{sec}$ photon flux with $1.2 \mathrm{MeV}$ photon energy.
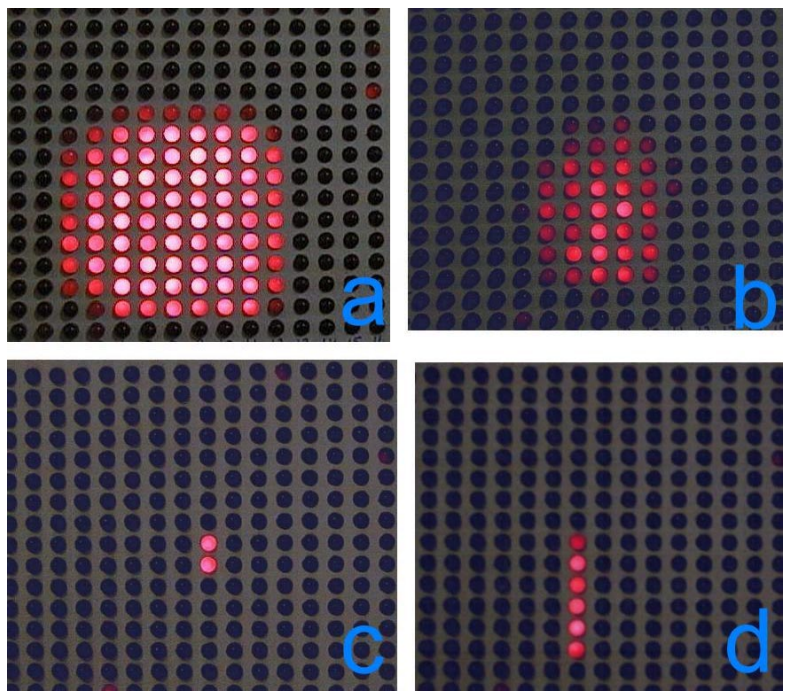

Fig. 4. Different images observed on the LED array with various undulator gap sizes and slits settings.

A 20-mm-thick tungsten slit was applied to form a narrow test beam 2.9-mm wide. The PSPCD sample was mounted on a precision stepping-motor-driven stage with linear encoder. The distance from the ${ }^{60} \mathrm{Co}$ source to the PSPCD was $480 \mathrm{~mm}$. The PSPCD test sample demonstrated a $0.059 \mathrm{nA} / \mathrm{mm}$ positioning sensitivity on a $10 \mathrm{~mm} \times 10 \mathrm{~mm}$ single element with 6 volts bias. Fig. 5 shows the PSPCD time response to the gamma-ray photon shutter. The PSPCD readout rise time was limited by the shutter speed and the system capacitance.

\section{SUMMARY}

We have developed a novel position-sensitive photoconductive detector using free-standing insulatingtype CVD diamond as its substrate material. Several different configurations, including 1-D and 2-D arrays as imaging detectors for beam profilers, have been developed. Tests on different PSPCD devices with highheat-flux undulator white beam, as well as with a highflux gamma-ray source, have been done at the APS and NIST. It was proven that the insulating-type CVD diamond can be used to make a hard x-ray positionsensitive detector based on the photoconductivity principle and that acts as a solid-state ion chamber.

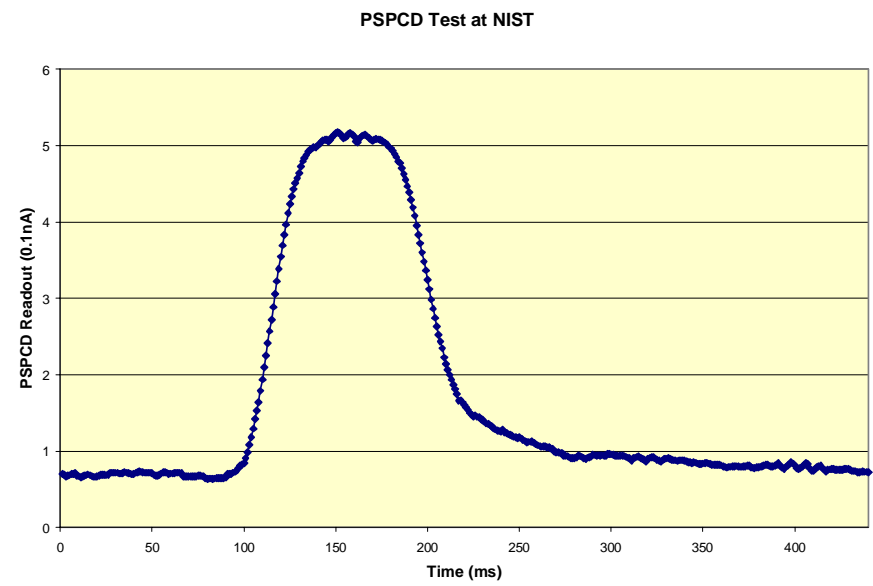

Fig. 5. PSPCD time response to the photon shutter motion during the test at the NIST high flux ${ }^{60} \mathrm{Co}$ gamma ray source.

\section{ACKNOWLEDGEMENTS}

The authors would like to thank Rogelio Ranay, Michel Lehmuller from the APS, and James Puhl from the NIST for their help in the PSPCD experiment. This work was supported by the U.S. Department of Energy, BES-Materials Science, under contract W-31-109-Eng38.

\section{REFERENCES}

[1]. Cotty W. F., (1956), Nature 177 1075-6.

[2]. Kozlov S. F., Stuck R., Hage-Ali M., and Siffert P., (1975), IEEE Trans. Nucl. Sci., NS-22 160-170.

[3]. Kania D. R., Pan L., Kornblum H., Bell P., Landen O. N., and Pianetta P., (1990), Rev. Sci. Instrum. 61 (10) 2765 .

[4]. Keddy R. J., Nam T. L., and Burns R. C., (1987), Phys. Med. Biol., Vol.32, No. 6, 751-759.

[5]. Kozlov S. F., Bachurin A. V., Petrusev S. S., and Fedorovsky Y. P., (1977), IEEE Trans. Nucl. Sci., NS-24 240-241.

[6]. D. Shu, and T.M. Kuzay, (1996), Rev. Sci. Instrum. 67(9).

[7]. D. Shu, J. Barraza, T. M. Kuzay, G. Naylor, and P. Elleaume, Proceedings of the 1997 International Particle Accelerator Conference, 2210 - 2213.

[8]. D. Shu, T. M. Kuzay, Y. Fang, J. Barraza, and T. Cundiff, (1998), Journal of Synchrotron Radiation, 5, 636-638. 